CrossMark

\title{
Lung transplantation after allogeneic stem cell transplantation: a pan-European experience
}

\author{
Mark Greer ${ }^{1,2}$, Cristina Berastegui ${ }^{3}$, Peter Jaksch ${ }^{4}$, Christian Benden $^{5}$, \\ John Aubert $\mathbb{0}^{6}$, Antoine Roux 7 , Elodie Lhuillier ${ }^{8}$, Sandrine Hirschi ${ }^{9}$, \\ Martine Reynaud-Gaubert ${ }^{10}$, François Philit ${ }^{11}$, Johanna Claustre ${ }^{12}$, \\ Pierre LePalud ${ }^{7}$, Marc Stern ${ }^{7}$, Christiane Knoop ${ }^{13}$, Robin Vos ${ }^{14}$, \\ Erik Verschuuren ${ }^{15}$, Andrew Fisher ${ }^{16}$, Gerdt Riise ${ }^{17}$, Lennart Hansson ${ }^{18}$, \\ Martin Iversen ${ }^{19}$, Pekka Hämmäinen ${ }^{20}$, Hans Wedel $^{21}$, Jacqueline Smits ${ }^{22}$, \\ Jens Gottlieb ${ }^{1,2}$ and Are M. Holm ${ }^{23,24,25}$
}

@ERSpublications

Lung transplantation is feasible in selected stem cell transplant recipients http://ow.ly/z4vn30h6gao

Cite this article as: Greer M, Berastegui C, Jaksch $\mathrm{P}$, et al. Lung transplantation after allogeneic stem cell transplantation: a pan-European experience. Eur Respir J 2018; 51: 1701330 [https://doi.org/10.1183/ 13993003.01330-2017].

ABSTRACT Late-onset noninfectious pulmonary complications (LONIPCs) affect $6 \%$ of allogeneic stem cell transplantation (SCT) recipients within 5 years, conferring subsequent 5 -year survival of $50 \%$. Lung transplantation is rarely performed in this setting due to concomitant extrapulmonary morbidity, excessive immunosuppression and concerns about recurring malignancy being considered contraindications. This study assesses survival in highly selected patients undergoing lung transplantation for LONIPCs after SCT.

SCT patients undergoing lung transplantation at 20 European centres between 1996 and 2014 were included. Clinical data pre- and post-lung transplantation were reviewed. Propensity score-matched controls were generated from the Eurotransplant and Scandiatransplant registries. Kaplan-Meier survival analysis and Cox proportional hazard regression models evaluating predictors of graft loss were performed.

Graft survival at 1,3 and 5 years of $84 \%, 72 \%$ and $67 \%$, respectively, among the 105 SCT patients proved comparable to controls $(\mathrm{p}=0.75)$. Sepsis accounted for 15 out of 37 deaths $(41 \%)$, with prior mechanical ventilation (HR 6.9, 95\% CI 1.0-46.7; $\mathrm{p}<0.001$ ) the leading risk factor. No SCT-specific risk factors were identified. Recurring malignancy occurred in four patients (4\%). Lung transplantation <2 years post-SCT increased all-cause 1-year mortality (HR 7.5, 95\% CI 2.3-23.8; $\mathrm{p}=0.001$ ).

Lung transplantation outcomes following SCT were comparable to other end-stage diseases. Lung transplantation should be considered feasible in selected candidates. No SCT-specific factors influencing outcome were identified within this carefully selected patient cohort.

This article has supplementary material available from erj.ersjournals.com

Received: July 032017 | Accepted after revision: Dec 012017

Conflict of interest: Disclosures can be found alongside this article at erj.ersjournals.com

Copyright @ERS 2018 
Affiliations: ${ }^{1}$ Hannover Medical School, Hannover, Germany. ${ }^{2}$ Biomedical Research in End-Stage and Obstructive Lung Disease (BREATH), German Center for Lung Research (DZL), Hannover, Germany. ${ }^{3}$ University Hospital Vall d'Hebron, Barcelona, Spain. "Medical University of Vienna, Vienna, Austria. ${ }^{5}$ University Hospital Zurich, Zurich, Switzerland. $U$ University Hospital Lausanne, Lausanne, Switzerland. ${ }^{7}$ Hospital Foch, Suresnes, France. ${ }^{8}$ Hospital Bichat, Paris, France. ${ }^{9}$ Civil Hospital, University of Strasbourg, Strasbourg, France. ${ }^{10}$ University North Hospital, Aix Marseille University, Marseille, France. ${ }^{11}$ Hospices Civils de Lyon, Lyon, France. ${ }^{12}$ University Hospital Grenoble, Grenoble, France. ${ }^{13}$ Erasme University Hospital, Brussels, Belgium. ${ }^{14}$ University Hospitals Leuven, Leuven, Belgium. ${ }^{15}$ University Medical Center, University of Groningen, Groningen, The Netherlands. ${ }^{16}$ Institute of Transplantation, Freeman Hospital and Newcastle University, Newcastle upon Tyne, UK. ${ }^{17}$ Sahlgrenska University Hospital, Gothenburg, Sweden. ${ }^{18}$ Skåne University Hospital, Lund, Sweden. ${ }^{19}$ Rigshospitalet, Copenhagen University Hospital, Copenhagen, Denmark. ${ }^{20}$ Helsinki University Hospital, Helsinki, Finland. ${ }^{21}$ Dept of Health Metrics, Sahlgrenska Academy, University of Gothenburg, Gothenburg, Sweden. ${ }^{22}$ Eurotransplant Foundation, Leiden, The Netherlands. ${ }^{23}$ Oslo University Hospital Rikshospitalet, Oslo, Norway. ${ }^{24}$ Institute of Clinical Medicine, University of Oslo, Oslo, Norway. ${ }^{25}$ Scandiatransplant, Aarhus, Denmark.

Correspondence: Mark Greer, Dept of Respiratory Medicine, OE6870, Hannover Medical School, Carl-Neuberg-Straße 1, 30625 Hannover, Germany. E-mail: greer.markamh-hannover.de

\section{Introduction}

Allogeneic haematopoietic stem cell transplantation (SCT) represents an established treatment option for an increasing number of benign and malignant diseases, with procedure rates exceeding 8000 in the USA and 14500 in Europe annually [1]. Leading indications include acute myeloid leukaemia (AML) and acute lymphocytic leukaemia (ALL), which currently account for $35 \%$ and $16 \%$ of procedures, respectively. Although refinements in SCT protocols have improved early outcomes, late-onset noninfectious pulmonary complications (LONIPCs) continue to limit long-term survival [2]. LONIPCs occur in $20 \%$ of SCT patients and encompass the entire spectrum of lung disease, ranging from interstitial lung disease, pleuroparenchymal fibroelastosis to pulmonary veno-occlusive disease and classical bronchiolitis obliterans [3]. Although the latter has long been considered ubiquitous for pulmonary graft versus host disease (GvHD), recent studies suggest additional interstitial lung involvement in some patients $[4,5]$. Treatment strategies ranging from oral macrolides to intensified immunosuppression and extracorporeal photopheresis have demonstrated benefit, but outcomes remain unpredictable and response rates disappointing [6-8].

Lung transplantation is considered viable treatment in carefully selected patients with various end-stage lung diseases. Since 1995, over 47000 adult lung transplantation procedures worldwide have been reported to the International Society for Heart and Lung Transplantation (ISHLT), with emphysema (38\%) being the leading indication [9]. Reports of lung transplantation for LONIPCs extend as far back as 1992 [10], but data is extremely limited. Of the 450 lung transplantations (1.1\%) performed for obliterative bronchiolitis in the past 20 years, only 65 were attributed to LONIPCs.

Shortages in suitable donor lungs universally limit lung transplant activity, with many regions adopting weighted scoring systems such as the Lung Allocation Score that focus on disease-related early survival probability, calculated from existing United Network for Organ Sharing registry data [11]. Inevitably, this discourages lung transplantation in orphan diseases along with conditions of poorer predicted outcome. Given SCT volume and the projected incidence of LONIPCs, lung transplantation rates are much lower than anticipated. Published experience is limited to case series and small cohorts with mixed outcomes, leading to a common perception that LONIPCs candidates are "high risk" [12-14], with perioperative sepsis due to excessive prior immunosuppression, complications of previous long-term steroid treatment, existing microbial colonisation and the ever-present risk of recurring, primary malignancy being cited as potential pitfalls [14].

The aim of this study is to quantify these risks and evaluate lung transplantation outcomes in carefully selected SCT patients with LONIPCs in a large multicentre cohort, paying particular attention to perioperative sepsis and recurring primary malignancy.

\section{Methods}

All patients undergoing lung transplantation for LONIPCs following allogeneic SCT at 20 European centres between 1996 and 2014 were analysed retrospectively. Clinical data pertaining to patient demographics, SCT treatment protocols, GvHD incidence and subsequent management were collated, along with imaging and native lung histology data prior to lung transplantation. Subsequent lung transplantation follow-up data until June 30, 2016 were standardised using detailed reporting protocols. 
Autologous SCT, living-donor lobar lung transplantation following SCT from the same donor and SCT patients with lung transplantation indications other than LONIPCs, such as smoking-related emphysema, were excluded. Life-long lung transplantation centre follow-up with a minimum of twice-yearly review was mandatory.

SCT were considered as being bone marrow transplantations or peripheral blood stem cell transplants using accepted definitions $[15,16]$.

Spirometry adhered to American Thoracic Society/European Respiratory Society guidelines and was performed at each attendance in all centres $[17,18]$. Baseline forced expiratory volume in $1 \mathrm{~s}\left(\mathrm{FEV}_{1}\right)$ and forced vital capacity (FVC) after lung transplantation were individually calculated from the mean of the two highest values recorded a minimum of 3 weeks apart. Diagnosis of chronic lung allograft dysfunction $(\mathrm{CLAD})$ required $\mathrm{a} \geqslant 3$-week reduction in $\mathrm{FEV}_{1}$ of $\leqslant 80 \%$ baseline in the absence of an alternative cause. Subclassification into obstructive bronchiolitis obliterans syndrome (BOS) or restrictive CLAD (rCLAD) adhered to currently proposed definitions [19-21].

A matched control cohort of non-SCT lung transplantation recipients was generated from the entire Eurotransplant and Scandiatransplant registries for first lung transplantation between 1996 and 2014 to compare lung transplantation graft survival. Propensity score matching for sex, age at lung transplantation, lung transplantation year, lung transplantation procedure (single or bilateral graft) and patient admission status at lung transplantation was performed using logistic regression with nearest-neighbour matching, a 0.2 calliper and a 1:50 matching ratio. Kaplan-Meier survival curves for overall graft as well as CLAD-free survival were constructed and the log-rank test used to assess significance.

Factors influencing 1-year mortality and early sepsis were assessed with single and multivariate Cox proportional hazard regression to calculate hazard ratios with $95 \%$ confidence intervals. The proportional hazards assumption was confirmed, taking $\mathrm{p}>0.05$ upon generation of the respective time-dependent covariates. Multivariate Cox regression analysis used forward stepwise (likelihood ratio) modelling, taking $\mathrm{p}=0.05$ and $\mathrm{p}=0.10$ as entry and removal cut-offs at each iteration.

Risk factors for 5-year recurrence of malignancy and 10-year CLAD development were assessed using competing risk regression, with missing values being omitted over a maximum 10 iterations.

Nonparametric tests were used for all variables and are reported as median (interquartile range (IQR)) unless otherwise stated. Categorical variables were tested with either the Chi-squared test or Fisher's exact test. Continuous variables were analysed using the Mann-Whitney U-test or Kruskal-Wallis H-test. Reported $\mathrm{p}$-values are two-tailed, with $\mathrm{p}<0.05$ being considered significant. Analysis was performed using SPSS Statistics for Macintosh version 24 (IBM, Armonk, NY, USA) and Prism version 6.0 (GraphPad, La Jolla, CA, USA).

\section{Results}

In total, 112 patients were identified. Seven were excluded from the analysis, with four considered to have had smoking-related emphysema and three due to autologous rather than allogeneic SCT. Of the 105 patients analysed, 66 (63\%) were male. Median (IQR) age at lung transplantation was 31.4 (20.543.5) years, with 18 out of 105 (17\%) being < 18 years old. AML was the leading SCT indication, comprising 38 out of 105 (36\%) patients. 12 patients had benign diseases, including thalassaemia, severe combined immunodeficiency, Wiskott-Aldrich syndrome and paroxysmal nocturnal haematuria. Demographic data are summarised in table 1.

All patients exhibited advanced lung disease with median FEV1 18\% and FVC 35\% predicted. Our previously reported clinical dichotomy in LONIPCs persisted [4]. Interstitial fibrosis was found in 51 out of 105 explanted lungs (49\%), corresponding to lower FVC (31\% versus $45 \%$ predicted; $\mathrm{p}=0.005)$ and TLC ( $89 \%$ versus $110 \%$ predicted; $\mathrm{p}=0.02)$ despite similar $\mathrm{FEV} 1$ ( $17 \%$ versus $18 \%$ predicted; $\mathrm{p}=0.58)$ in those patients.

In the absence of specific guidelines for lung transplantation candidate selection for patients post-SCT, 17 out of 20 centres (85\%) reported adapting the ISHLT consensus for cystic fibrosis (colonisation, body mass index) in conjunction with a 2-year cancer-free interval prior to listing [23]. Otherwise no additional criteria were reported. In the three remaining centres, formal multidisciplinary collaboration with local haematologists was undertaken to assure quiescent extrapulmonary GvHD. All centres reported monitoring serum immunoglobulins prior to transplant, with substitution only being offered to those demonstrating IgG deficiency in the setting of two or more episodes of infection within 6 months.

During both the peri- and post-lung transplantation phases, considerable variation in standard management across the 20 centres was evident, due in part to variations in local reimbursement policies 
TABLE 1 Summary of stem cell transplantation (SCT) patient demographics, including treatment up to and including lung transplantation

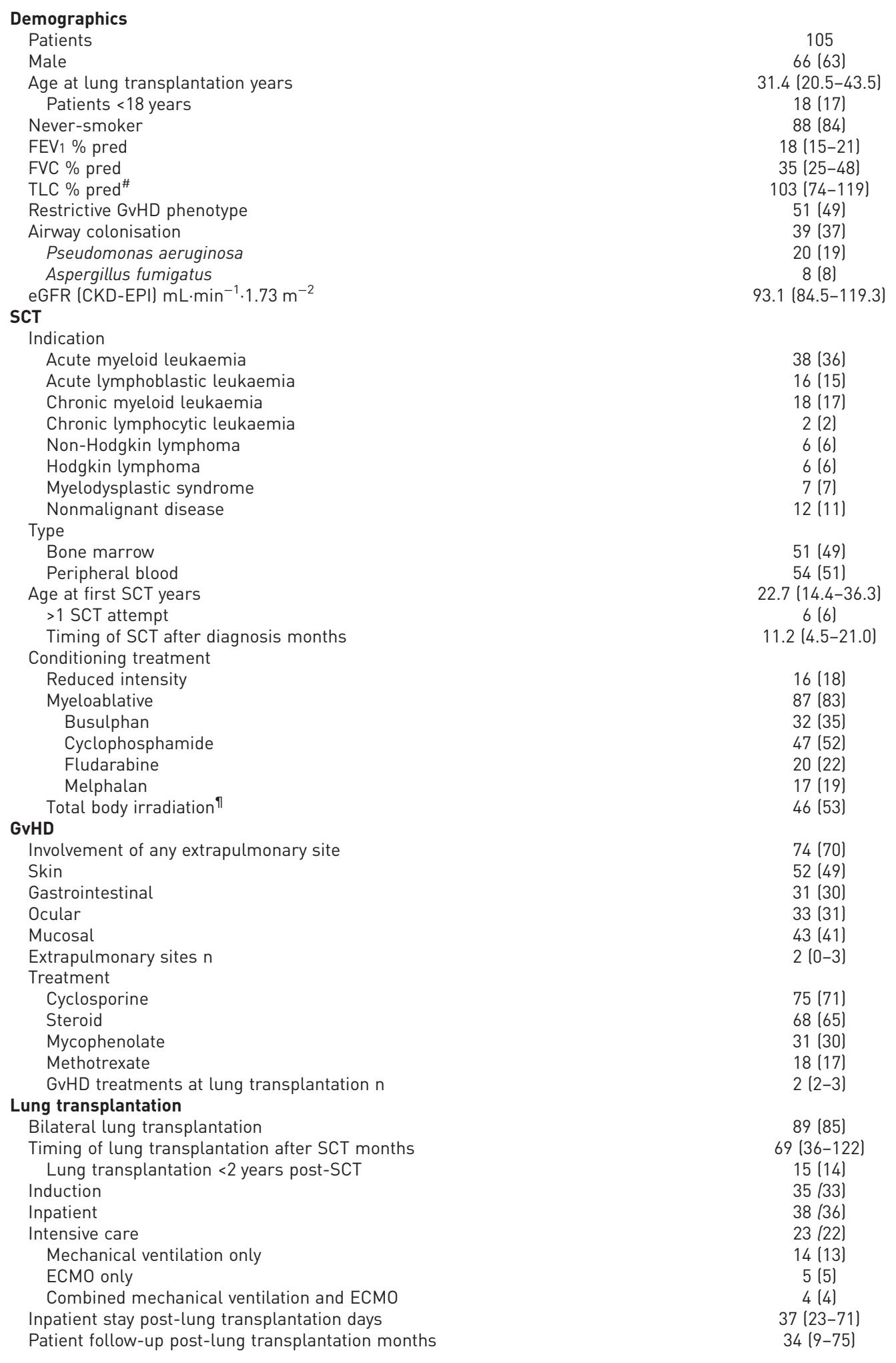

Data are presented as $\mathrm{n}, \mathrm{n}(\%)$ or median (interquartile range). FEV1: forced expiratory volume in $1 \mathrm{~s} ; \mathrm{FVC}$ : forced vital capacity; TLC: total lung capacity; GvHD: graft versus host disease; eGFR (CKD-EPI): estimated glomerular filtration rate (calculated using the Chronic Kidney Disease Epidemiology Collaboration (2012) formula [22]); ECMO: extracorporeal membrane oxygenation. ${ }^{\#}: \mathrm{n}=91$ patients; ${ }^{\text {ๆ }}$ : n=86 patients. 
across the 12 jurisdictions involved. Induction therapy was routinely used in seven out of 20 centres (35\%), accounting for 35 out of 105 SCT patients (33\%). All but one centre used the same agent as for non-SCT patients. The remaining centre replaced their standard antithymocyte globulin with basiliximab due to physician preference. In total, 21 out of 105 patients (20\%) received basiliximab and 14 out of 105 patients (13\%) received antithymocyte globulin. Neither receipt of induction therapy nor either of the specific agents used appeared to influence the risk of sepsis (HR 0.83, 95\% CI $0.21-3.29 ; \mathrm{p}=0.79$ ) or early mortality (HR 0.30, 95\% CI 0.04-2.50; $\mathrm{p}=0.27$ ).

All centres initiated standard triple immunosuppression post-lung transplantation, combining a calcineurin inhibitor, antimetabolite and steroids. The commonest protocol was tacrolimus, mycophenolate and prednisolone, which was used in 79 out of 105 patients (75\%). Cyclosporine A was used in the remaining 26 patients, seven of whom also received azathioprine instead of mycophenolate as per standard local policy. Prednisolone dosage of $\leqslant 10 \mathrm{mg} \cdot \mathrm{day}^{-1}$ at 4 weeks post-lung transplantation was targeted at all institutions.

Anti-infective prophylaxis again did not deviate between SCT and non-SCT patients at individual centres, but significant variation between institutional protocols existed. Life-long Pneumocystis jirovecii prophylaxis along with fungal prophylaxis in the first 4 weeks was advocated by all centres. Life-long systemic azole prophylaxis was offered to only 28 out of 105 patients (27\%). The remaining centres reported aggressive management of Aspergillus species if detected during surveillance. Reported rates of life-threatening fungal infections among the SCT group was low, with only a single fatality reported. Cytomegalovirus prophylaxis again followed local protocols for all patients, varying between 4 weeks to 12 months depending on the donor and recipient constellation.

Total lung transplantation volume at the participating centres during the observation period was 10693 , with current centre volumes ranging from 15 to 120 procedures per year (median 40 per year). Median (IQR) patient follow-up post-lung transplantation was 34 (9-75) months.

\section{Graft survival}

Graft survival at 1, 3 and 5 years post-lung transplantation was $85 \%, 72 \%$ and $67 \%$, respectively. 37 patients (35\%) died, with sepsis and CLAD being the leading causes of death.

Within Eurotransplant and Scandiatransplant, 9895 first-time lung transplantations were performed between 1996 and 2014. Using the defined criteria, 4075 patients were included in the matched control cohort (supplementary table S1). Given the comparatively young age and high rates of bilateral lung transplantation in the SCT cohort, it should be noted that cystic fibrosis (CF) and non-CF bronchiectasis were disproportionately over-represented ( $32 \%$ versus $18 \%$ of the registry cohort) and emphysema correspondingly under-represented ( $29 \%$ versus $41 \%)$. Compared with controls, no differences in graft survival at 1,3 or 5 years were evident (figure 1).

16 patients (15\%) died within 12 months, of whom 14 did not survive to hospital discharge. Sepsis accounted for 10 of these deaths. In the univariate Cox regression analysis, the only SCT-specific risk factors to achieve significance were prior calcineurin inhibitor treatment within 6 months of lung transplantation and a time interval of $<2$ years between the final SCT and lung transplantation (table 2). However, only the latter retained significance in the subsequent multivariate analysis (HR 7.5, 95\% CI 2.323.8; $\mathrm{p}=0.001$ ), along with $\mathrm{FEV} 1 / \mathrm{FVC} \geqslant 0.7$ (HR 5.0, 95\% CI 1.4-17.9; $\mathrm{p}=0.012$ ) and requiring inpatient care immediately prior to lung transplantation (HR 3.4, 95\% CI 1.2-10.0; $\mathrm{p}=0.03$ ).

\section{Sepsis}

In total, 15 out of 37 deaths (41\%) during the observation period were attributable to sepsis. Three deaths occurred in patients with advanced CLAD exhibiting the BOS phenotype. Eight of the 12 remaining deaths occurred perioperatively, with median (IQR) survival 42 (24-67) days. Other than a single case of fatal cytomegalovirus infection within 90 days, no details regarding the fatal pathogens in the remaining cases were available. Only two had reported colonisation prior to lung transplantation (Pseudomonas aeruginosa and methicillin-sensitive Staphylococcus aureus, respectively).

Univariate Cox regression identified ALL as the sole SCT risk factor for early sepsis (HR 4.6, 95\% CI 1.318.7; $\mathrm{p}=0.02$ ), alongside deteriorating native lung and kidney function and increasing dependency on medical support immediately prior to transplant (supplementary table S2). In the multivariate analysis, only the latter retained significance with intensive care unit (ICU) admission prior to lung transplantation (HR 5.2, 95\% CI 1.2-23.4; $\mathrm{p}=0.03$ ), mechanical ventilation (HR 6.9, 95\% CI 1.0-46.7; $\mathrm{p}<0.001$ ) and dual bridging (HR 7.8, 95\% CI 6.2-98.3; p=0.001) being relevant. 


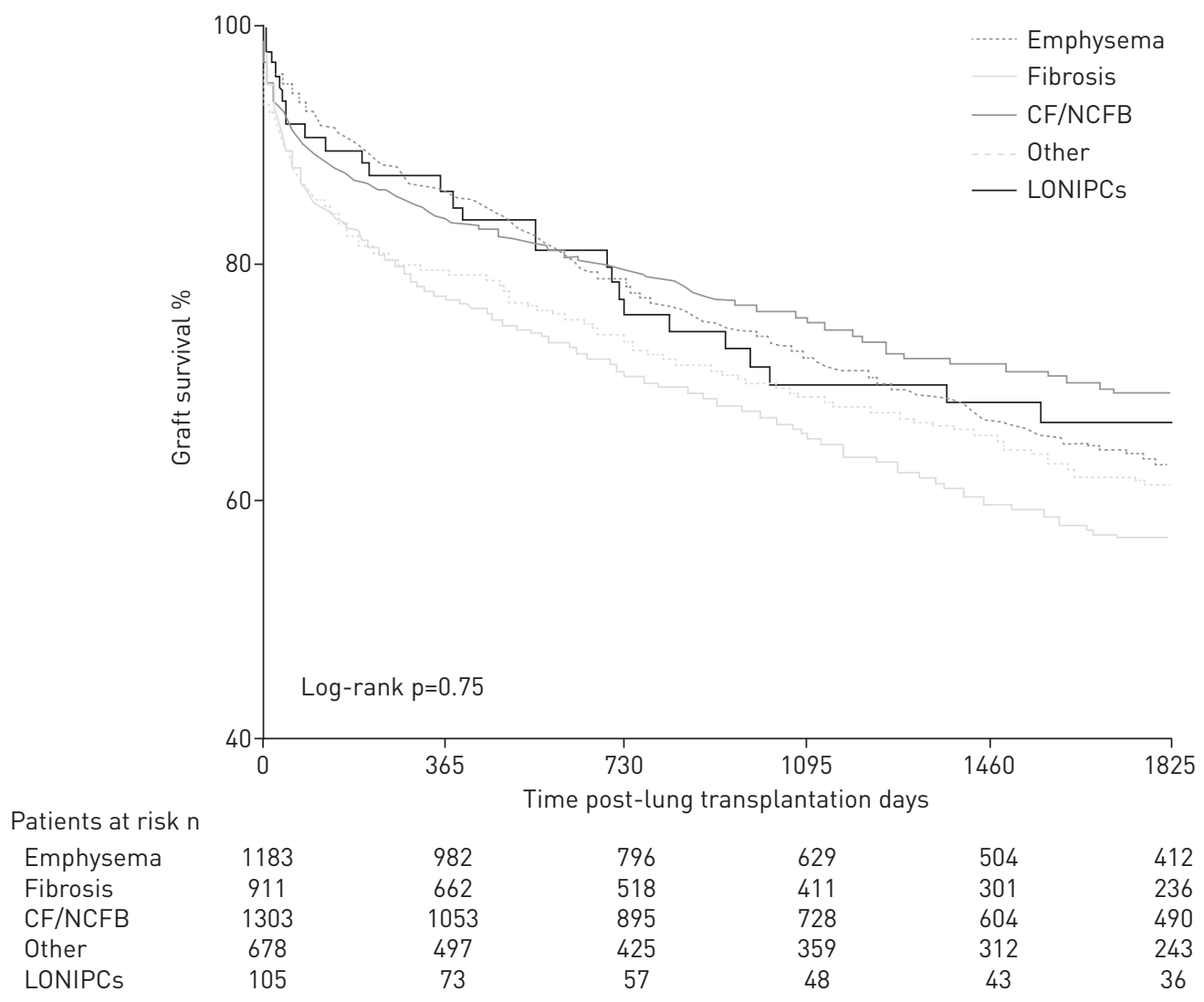

FIGURE 1 Kaplan-Meier curve showing graft survival following first lung transplantation among allogeneic stem cell transplantation (SCT) patients as well as matched controls from the other main disease groups. CF: cystic fibrosis; NCFB: non-CF bronchiectasis; LONIPCs: late-onset noninfectious pulmonary complications after SCT. Comparing SCT versus non-SCT patients, no differences in survival were observed at 1, 3 and 5 years post-lung transplantation.

\section{Malignancy}

Malignancy accounted for eight out of 37 deaths (22\%), with all occurring among the 93 patients with malignant SCT indications. However, only half were due to recurrence of the original malignancy. AML accounted for two cases in whom recurrence occurred within 12 months of lung transplantation, with death ensuing within 12 months of diagnosis. Both patients had received lung transplantation within 2 years of SCT, well below the median (IQR) interval of 72 (47-135) months in the other 34 AML patients.

Single recurrences of ALL and non-Hodgkin lymphoma were recorded, with both presenting much later at 3 and 5 years post-lung transplantation, respectively. Competing risks regression analysis for recurrence within 5 years of lung transplantation suggested both myeloablative induction therapy at SCT and lung transplantation within 2 years of SCT as being contributory.

The four remaining fatal malignancies were colorectal cancer, melanoma and two cases of post-transplantation lymphoproliferative disorder unrelated to the primary malignancy. Taking all eight malignancies together, the only significant risk factor on multivariate Cox regression was lung transplantation within 24 months of SCT (HR 6.4, 95\% CI 1.3-46.0; $\mathrm{p}=0.03$ ).

\section{Extrapulmonary chronic GvHD}

Chronic GvHD involving other organs was reported in 74 out of 105 patients (70\%), with skin involvement exhibiting the highest prevalence (49\%) and gastrointestinal disease the lowest $(30 \%)$ (table 1). Within the cohort, neither involvement of specific organs $(\mathrm{p}=0.88)$ nor cumulative site involvement $(\mathrm{p}=0.95)$ impacted on lung transplantation survival. Notably, inpatient lung transplantation had lower rates of extrapulmonary GvHD (27\% versus 55\%; $\mathrm{p}=0.007)$, particularly skin involvement ( $25 \%$ versus $45 \% ; \mathrm{p}=0.03)$. No differences in incidence regarding ICU or bridging requirements were observed. 
TABLE 2 Uni- and multivariate Cox regression analyses assessing risk factors for all-cause mortality $\leqslant 12$ months after lung transplantation

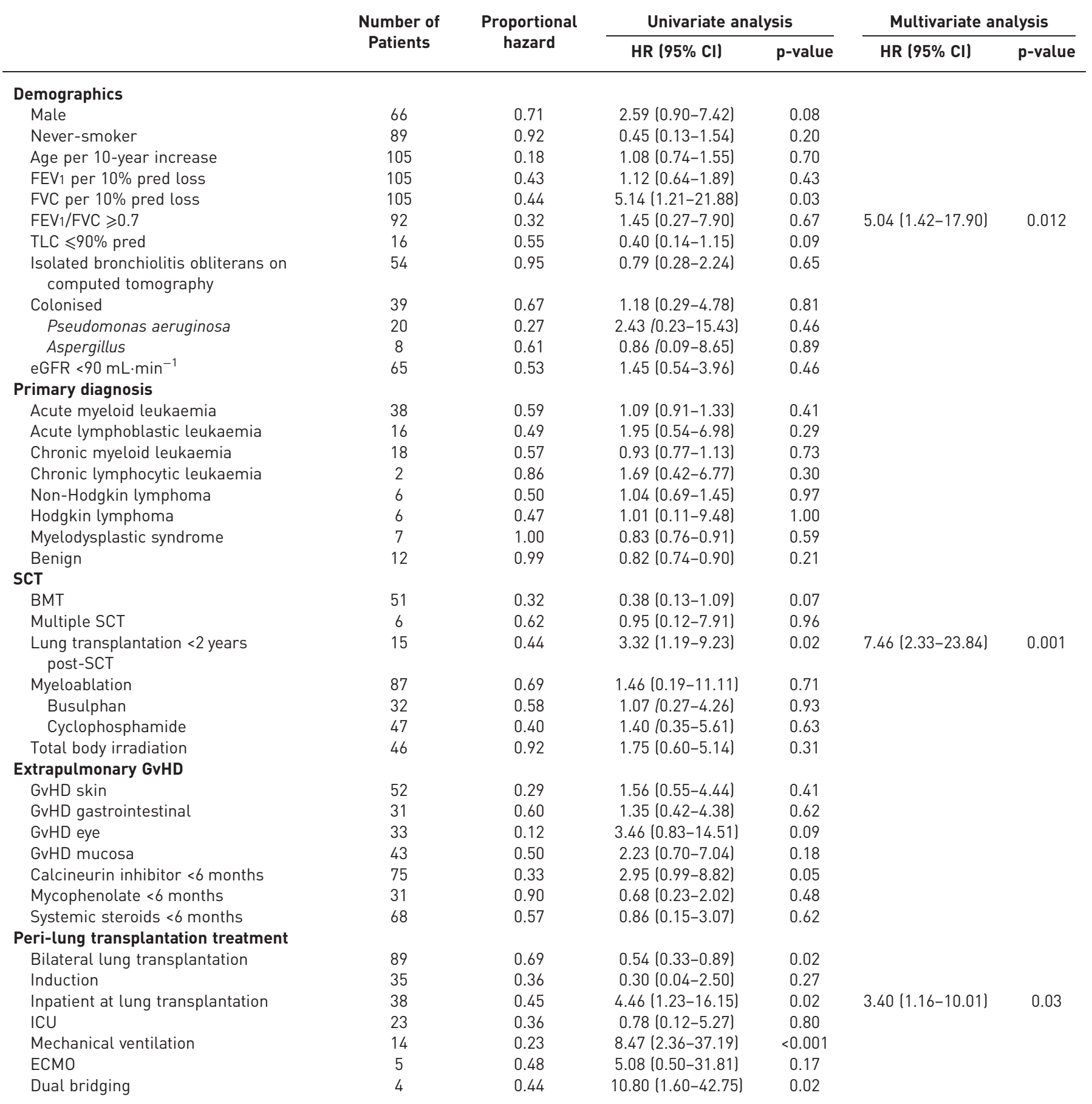

FEV1: forced expiratory volume in $1 \mathrm{~s}$; FVC: forced vital capacity; TLC: total lung capacity; eGFR: estimated glomerular filtration rate; SCT: stem cell transplantation; BMT: bone marrow transplantation; GvHD: graft versus host disease; ICU: intensive care unit; ECMO: extracorporeal membranous oxygenation.

\section{CLAD and re-transplantation}

CLAD developed in 29 out of 79 patients (37\%) surviving beyond the first year, developing at median (IQR) 29 (15-56) months post-lung transplantation. Both CLAD phenotypes were evident, with 21 out of $29(72 \%)$ developing BOS and the remaining eight out of $29(28 \%)$ developing rCLAD. No clear relationship between CLAD onset or phenotype and previous LONIPC phenotype was evident. rCLAD 


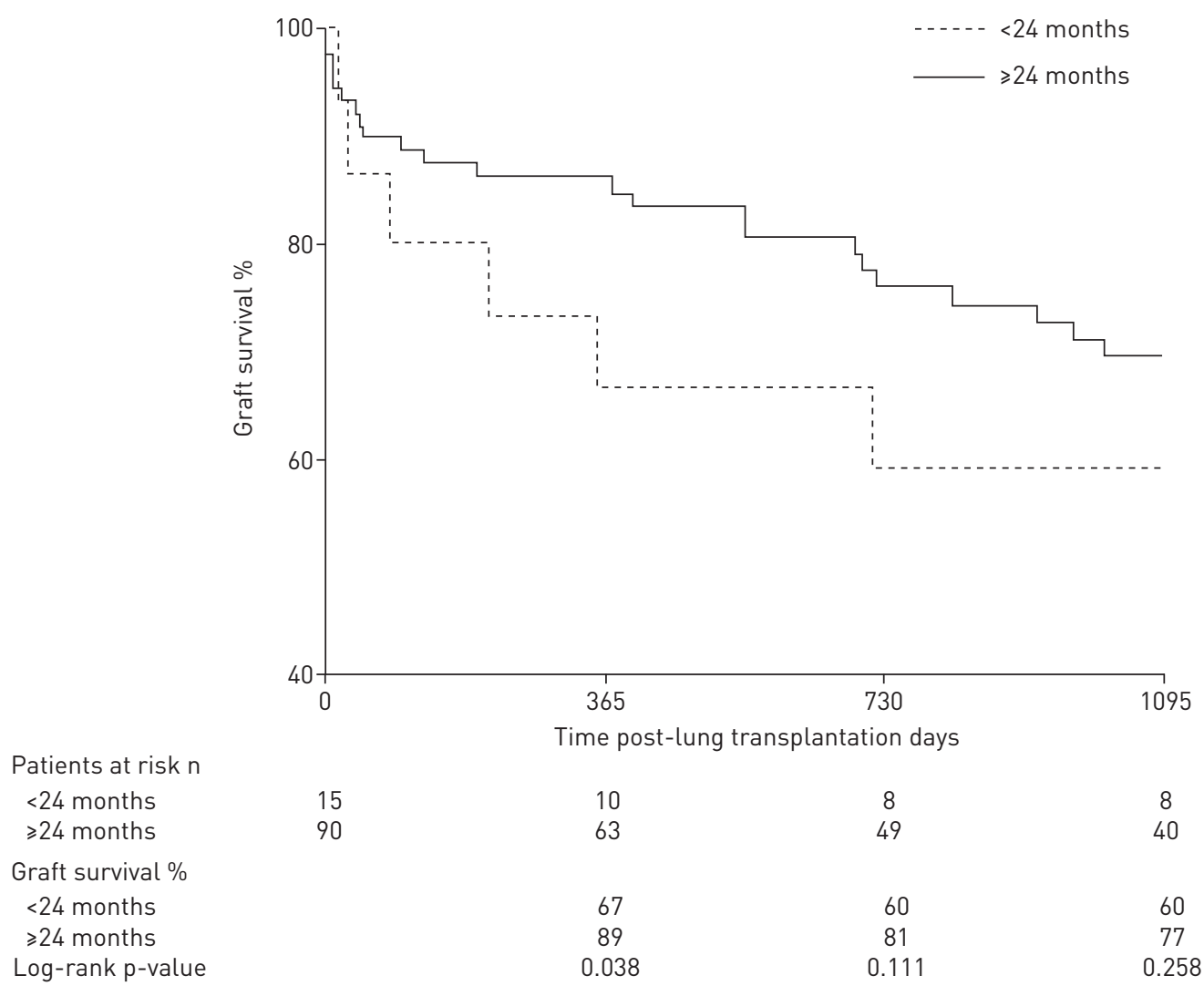

FIGURE 2 Kaplan-Meier curve showing graft survival following first lung transplantation among allogeneic stem cell transplantation (SCT) patients, dependent upon the time interval between procedures $\mid<24$ or $\geqslant 24$ months). Early survival was worse in those proceeding to lung transplantation within 2 years of SCT, but beyond the first year no differences remained.

occurred earlier (median 13 versus 47 months; $\mathrm{p}=0.002$ ) and resulted in earlier graft loss (median 30 versus 71 months post-lung transplantation; $\mathrm{p}=0.01$ ). Limitations of the registry data used for the matched control cohort unfortunately prevented accurate comparisons for CLAD-free and with-CLAD survival. Broad comparisons with ISHLT data for CLAD-free survival, dependent upon surviving $\geqslant 14$ days post-lung transplantation, appear favourable (median 5.6 versus 11.9 years), but interpretation is limited.

Competing risks regression analysis for 10 -year CLAD risk identified only need for ICU $(p=0.02)$ and mechanical ventilation $(p=0.01)$, with no evidence of any immunological associations between CLAD phenotype and previous GvHD characteristics.

Five redo lung transplantations were performed. Two were attempted for primary graft dysfunction, with both patients dying within 1 week. CLAD accounted for the remaining procedures, of which two had rCLAD. One rCLAD patient died perioperatively due to bleeding. The remaining two redo lung transplantation patients remained alive at study completion, 12 and 20 months post-redo transplant, respectively.

\section{Discussion}

This pan-European experience of lung transplantation in carefully selected candidates with life-limiting LONIPCs following allogeneic SCT represents the largest known cohort of such patients. The most significant finding was that survival at 1, 3 and 5 years in this cohort was similar to matched controls with other end-stage lung diseases.

Given the current reported SCT volumes and reported incidence and outcomes of LONIPCs, approximately 350 patients die annually in Europe alone. Demonstrating the feasibility of lung transplantation and generating awareness is important for both pulmonologists and haematologists alike. Although it is unlikely that all would be suitable lung transplantation candidates, the disparity to current lung transplantation rates remains hard to ignore. 
These findings provide encouraging guidance to lung transplantation physicians, assisting in difficult decision making regarding candidacy of such patients. Discouragement of orphan diseases is an inherent limitation of organ allocation systems. With the intent of optimising graft utility, their reliance on 1-year outcome data tends to discriminate when a critical mass of data does not exist. Furthermore, the body of evidence available appears to suggest that such carefully selected patients can be managed in a very similar fashion to other lung transplantation recipients, without affecting outcomes.

Being a retrospective analysis involving numerous centres of varying lung transplantation experience, it is inevitable given the condition's reputation that cautious decision making resulted in candidate selection bias at listing. Young recipient age, high outpatient lung transplantation rates and lack of comorbidities among hospitalised patients are all suggestive. Ideally, consideration of rejected candidate referrals and wait-list deaths would add value to the analysis, but obtaining data from all centres proved unfeasible. While minimising interobserver variability, use of standardised data collection enforced a "lowest common denominator" approach to maximise completeness, generating more qualitative data. For certain aspects, such as treatment exposure or extent of disease, greater reliance on quantitative data may have proved more insightful.

Due caution is encouraged in interpreting the Cox proportional hazard models given the infrequent events within a small cohort. The spread in the $95 \%$ confidence intervals reflect this, casting a degree of uncertainty that can only be resolved with increasing case volume. As such, the results reflect current experience to assist physicians facing difficult decision making when assessing or managing such patients.

Nonetheless, the findings provide an important insight into the factors impacting upon early survival, and dispel some of the common concerns about sepsis risk and recurring malignancy. The relevance of 1-year all-cause mortality is rooted in allocation policies. Apart from lung transplantation within 2 years of SCT, all the identifiable risk factors in the uni- and multivariate analyses reflected the general clinical condition and, as highlighted in the ISHLT Registry, are by no means exclusive to LONIPCs [9]. Interestingly, the recent recognition of LONIPC phenotypes appears relevant, as those with restrictive spirometry (FEV1/ FVC $\geqslant 70 \%$ : HR 5.0, 95\% CI 1.4-17.9; p=0.012) exhibited poorer early survival on multivariate analysis. However, it is unclear if this reflects patient condition or the duration of prior immunosuppression which, as stated previously [4], was significantly longer among patients with restrictive lung function (median (IQR) 54 (30-108) versus 114 (70-152) months; $\mathrm{p}=0.002)$.

Early fatal sepsis accounted for eight out of 17 deaths (47\%), which appears to exceed (192 out of 781 deaths (25\%)) ISHLT Registry reports [9]. Except for ALL, no other SCT-specific factors were identified. Caution due to the small numbers involved is again advised, but four out of 16 lung transplantation recipients with a history of ALL died of early sepsis, accounting for half of such deaths. No clear explanation was evident, but it is noteworthy that sepsis-related death is problematic among ALL patients following SCT, with immunoglobulin deficiencies being implicated [24, 25]. Importantly, however, concerns related to prior immunosuppression or microbial airway colonisation could not be substantiated. The consensus view among the authors was that colonisation should not represent an absolute contraindication, rather remain an individual case consideration. It should be noted, however, that none of the cohort were colonised with more contentious pathogens such as Mycobacterium abscessus, Scedosporium or Burkholderia species.

Recurrence rates of primary malignancy were low at $4 \%$. Given the small numbers involved and proportionately large number of early deaths of alternative cause, concealed confounders are likely. A competing risks regression was used to compensate for this, identifying lung transplantation within 2 years of last SCT as the only risk factor. While AML data appear to support this, the interaction between factors remains unclear and should be considered in context. Current candidate selection guidelines recommend a malignancy-free interval of 2 years for all lung transplantation [23] and given the all-cause mortality data presented here (figure 2), no clear evidence to the contrary can be provided.

Extrapulmonary chronic GvHD causes considerable comorbidity and its lack of influence on outcomes suggests inadequacy of data. Concerns regarding inadequate nutrition or oral immunosuppression in gastrointestinal GvHD should be considered analogous to those in CF where attainment of stable body mass with or without enteral feeding represents a basic lung transplantation requirement [23].

Given their similarities, both in terms of end-organ damage and unpredictable responses to broadly similar treatment strategies, the development of host versus graft CLAD in patients with existing GvHD disease is somewhat unique. Unfortunately, no associations regarding incidence, timing or phenotypic recurrence were found. Furthermore, there was nothing in the observed CLAD demographics to suggest substantial deviation from reported patterns in other disease groups. 
In conclusion, lung transplantation is a feasible treatment option for refractory LONIPCs in thoroughly evaluated, carefully selected candidates, with survival rates comparable to other indications. Accurate characterisation of LONIPCs and timely referral appear important in minimising risk. Candidate evaluation, selection and perioperative care should adhere to similar local protocols and values afforded to other end-stage lung diseases.

\section{Acknowledgements}

Author contributions: Study design, data collection, analysis, interpretation and preparation of the manuscript: M. Greer, J. Gottlieb and A. Holm. Statistical analysis: M. Greer and H. Wedel. Data collection and critical revision of the manuscript: C. Berastegui, P. Jaksch, C. Benden, J. Aubert, A. Roux, E. Lhuillier, S. Hirschi, M. Reynaud-Gaubert, F. Philit, J. Claustre, P. LePalud, M. Stern, C. Knoop, R. Vos, E. Verschuuren, A. Fisher, G. Riise, L. Hansson, M. Iversen, P. Hämmäinen and J. Smits.

\section{References}

1 Passweg JR, Baldomero H, Bader P, et al. Hematopoietic SCT in Europe 2013: recent trends in the use of alternative donors showing more haploidentical donors but fewer cord blood transplants. Bone Marrow Transplant 2015; 50: 476-482.

2 Gooley TA, Chien JW, Pergam SA, et al. Reduced mortality after allogeneic hematopoietic-cell transplantation. N Engl J Med 2010; 363: 2091-2101.

3 Bergeron A. Late-onset noninfectious pulmonary complications after allogeneic hematopoietic stem cell transplantation. Clin Chest Med 2017; 38: 249-262.

4 Greer M, Riise GC, Hansson L, et al. Dichotomy in pulmonary graft-versus-host disease evident among allogeneic stem-cell transplant recipients undergoing lung transplantation. Eur Respir J 2016; 48: 1807-1810.

5 Filipovich $\mathrm{AH}$, Weisdorf D, Pavletic S, et al. National Institutes of Health consensus development project on criteria for clinical trials in chronic graft-versus-host disease: I. Diagnosis and staging working group report. Biol Blood Marrow Transplant 2005; 11: 945-956.

6 Radhakrishnan SV, Palaniyandi S, Mueller G, et al. Preventive azithromycin treatment reduces noninfectious lung injury and acute graft-versus-host disease in a murine model of allogeneic hematopoietic cell transplantation. Biol Blood Marrow Transplant 2015; 21: 30-38.

7 Cutler C, Logan B, Nakamura R, et al. Tacrolimus/sirolimus vs tacrolimus/methotrexate as GVHD prophylaxis after matched, related donor allogeneic HCT. Blood 2014; 124: 1372-1377.

8 Lucid CE, Savani BN, Engelhardt BG, et al. Extracorporeal photopheresis in patients with refractory bronchiolitis obliterans developing after allo-SCT. Bone Marrow Transplant 2011; 46: 426-429.

9 Yusen RD, Edwards LB, Kucheryavaya AY, et al. The Registry of the International Society for Heart and Lung Transplantation: Thirty-second Official Adult Lung and Heart-Lung Transplantation Report - 2015; Focus Theme: Early Graft Failure. J Heart Lung Transplant 2015; 34: 1264-1277.

10 Calhoon JH, Levine S, Anzueto A, et al. Lung transplantation in a patient with a prior bone marrow transplant. Chest 1992; 102: 948.

11 Egan TM, Edwards LB. Effect of the lung allocation score on lung transplantation in the United States. $J$ Heart Lung Transplant 2016; 35: 433-439.

12 Vogl UM, Nagayama K, Bojic $M$, et al. Lung transplantation for bronchiolitis obliterans after allogeneic hematopoietic stem cell transplantation: a single-center experience. Transplantation 2013; 95: 623-628.

13 Holm AM, Riise GC, Hansson L, et al. Lung transplantation for bronchiolitis obliterans syndrome after allo-SCT. Bone Marrow Transplant 2013; 48: 703-707.

14 Koenecke C, Hertenstein B, Schetelig J, et al. Solid organ transplantation after allogeneic hematopoietic stem cell transplantation: a retrospective, multicenter study of the EBMT. Am J Transplant 2010; 10: 1897-1906.

15 Korbling M, Anderlini P. Peripheral blood stem cell versus bone marrow allotransplantation: does the source of hematopoietic stem cells matter? Blood 2001; 98: 2900-2908.

16 Thomas ED, Storb R. Technique for human marrow grafting. Blood 1970; 36: 507-515.

17 Pellegrino R, Viegi G, Brusasco V, et al. Interpretative strategies for lung function tests. Eur Respir J 2005; 26 : 948-968.

18 Miller MR, Hankinson J, Brusasco V, et al. Standardisation of spirometry. Eur Respir J 2005; 26: 319-338.

19 Verleden GM, Raghu G, Meyer KC, et al. A new classification system for chronic lung allograft dysfunction. J Heart Lung Transplant 2014; 33: 127-133.

20 Estenne M, Maurer JR, Boehler A, et al. Bronchiolitis obliterans syndrome 2001: an update of the diagnostic criteria. J Heart Lung Transplant 2002; 21: 297-310.

21 Verleden SE, Ruttens D, Vandermeulen E, et al. Restrictive chronic lung allograft dysfunction: where are we now? $J$ Heart Lung Transplant 2015; 34: 625-630.

22 Inker LA, Schmid $\mathrm{CH}$, Tighiouart $\mathrm{H}$, et al. Estimating glomerular filtration rate from serum creatinine and cystatin C. N Engl J Med 2012; 367: 20-29.

23 Weill D, Benden C, Corris PA, et al. A consensus document for the selection of lung transplant candidates: 2014 - an update from the Pulmonary Transplantation Council of the International Society for Heart and Lung Transplantation. J Heart Lung Transplant 2015; 34: 1-15.

24 O'Connor D, Bate J, Wade R, et al. Infection-related mortality in children with acute lymphoblastic leukemia: an analysis of infectious deaths on UKALL2003. Blood 2014; 124: 1056-1061.

25 Hung IJ, Yang CP. Early-onset sepsis in children with acute lymphoblastic leukemia. J Formos Med Assoc 1996; 95: 746-753. 